\title{
EPIDEMIOLOGÍA DE LA INCONTINENCIA URINARIA EN EL CÁNCER DE PRÓSTATA. INCIDENCIA, CALIDAD DE VIDA Y ASPECTOS FARMACOECONÓMICOS
}

\author{
Anna Orsola y Juan Morote.
}

Servicio de Urología. Hospital Vall d'Hebrón. Barcelona. España.

\begin{abstract}
Resumen.- OBJETIVO: Presentar datos recientes sobre la epidemiología de la incontinencia urinaria en el cáncer de próstata (CaP). Asimismo hacer un repaso de su incidencia, del impacto en la calidad de vida y de los aspectos farmacoeconómicos relacionados.

MÉTODO: Se ha realizado una revisión de la literatura referente a las complicaciones de los distintos métodos de tratamiento del CaP incluyendo la prostatectomia radical -PR- (abierta, laparoscópica y robótica), la radioterapia externa, la braquiterapia, la crioterapia y los ultrasonidos de alta-intensidad (high-intensity focused ultrasound -HIFU-).
\end{abstract}

RESULTADOS: La falta de uniformidad en la definición de incontinencia, la manera de evaluarla y la manera de reportarla hacen que la interpretación de los resultado funcionales y del impacto en la calidad de vida tras cualquiera de los tratamientos del CaP sea dispar y difi-

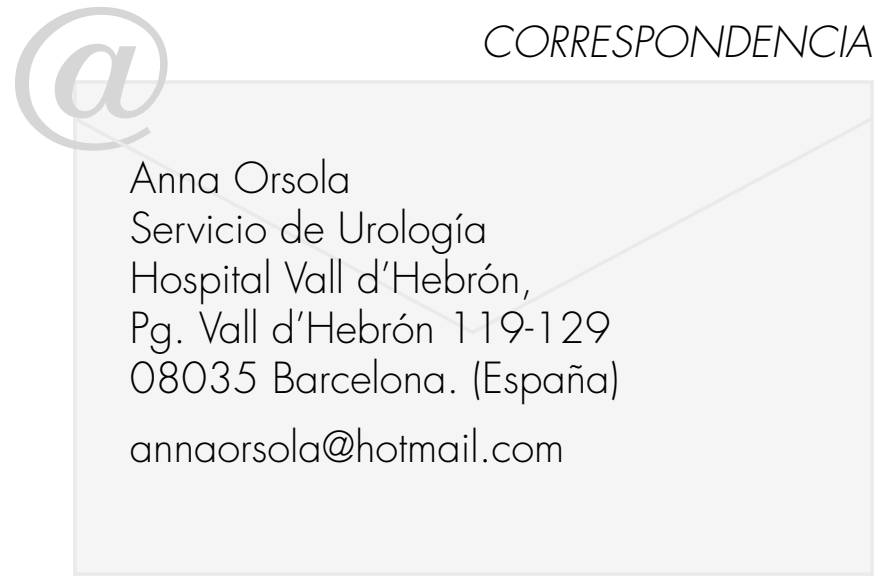

cil de interpretar. En líneas generales se ha documentado que la incontinencia urinaria post tratamiento afecta con mayor frecuencia a los pacientes tratados mediante cirugía radical. Sin embargo, se constata también que la preservación de bandeletas neurovasculares, los realización de los procedimientos en hospitales con gran volumen de pacientes y por cirujanos con un elevado número de casos y el desarrollo de la PR robótica parecen afectar positvamente el rendimiento global de la técnica. En la radioterapia externa, la incontinencia moderada es menos frecuente pero la asociación de dolor rectal y diarrea, que llegan a desarrollar hasta el 40\% de los pacientes, agrava la sintomatología miccional. En la braquiterapia transperineal los síntomas irritativos y obstructivos se asocian en especial a disfunción eréctil desarrollada a largo plazo así como a morbilidad rectal. En el caso de la crioterapia y del HIFU los estudios son series de casos y no hay estudios randomizados que comparen estas terapias como tratamiento primario del CaP localizado.

CONCLUSIONES: Cada modalidad de tratamiento para el CaP se asocia a un patrón distinto de cambios en los dominios de calidad de vida relacionados con la función urinaria, sexual, intestinal y hormonal. Dos factores son claves cuando se evalúa la incontinencia; la documentación del grado de continencia previa al procedimiento y el uso de métodos de evaluación validados y autoadministrados. Aunque las mejoras técnicas en todos los procedimientos deberían contribuir a disminuir el impacto de las complicaciones, no hay que olvidar la tendencia a asociar tratamientos -terapias multimodales- tienen un perfil de complicaciones mas elevado. Por lo que deben reservarse para aquellos pacientes en los que se haya demostrado un beneficio.

Palabras clave: Incontinencia urinaria. Cáncer de próstata. Calidad de vida. 
Summary.- OBJECTIVES: To present recent data on the epidemiology of urinary incontinence in prostate cancer (PCa). To review the incidence of urinary incontinence, its impact on quality of life and related pharmacoeconomic features.

METHODS: We performed a bibliographic review about the complications of the various therapeutic options for PCa including radical prostatectomy (RP) lopen, laparoscopic and roboticl, external beam radiotherapy, brachytherapy, cryotherapy, and high intensity focused ultrasound (HIFU).

RESULTS: The lack of uniformity for urinary incontinence definition, for its evaluation, and for the way to report it makes the interpretation of functional results and impact on quality of life after any treatment option difficult and uneven. Generally, we documented that urinary incontinence after treatment appears more often in patients undergoing radical surgery. Nevertheless, we stated that neurovascular bundle preservation, performance of the procedure in high volume centers, by high volume surgeons, and development of robotic surgery may positively influence the global outcomes of this technique. Moderate incontinence is less frequent after external beam radiotherapy, but the association of rectal pain and diarrhea, in up to $40 \%$ of the patients, worsens voiding symptoms. Irritative and obstructive voiding symptoms after perineal brachytherapy are especially associated with long term erectile dysfunction as well as rectal morbidity. In the case of cryotherapy and HIFU the available studies are case series and there are not randomized studies comparing them with the primary treatment of localized PCa.

CONCLUSIONS: Each treatment modality for PCa is associated with a different pattern of changes in the urinary, sexual, intestinal and hormonal related quality of life domains. Two key factors when evaluating incontinence are information about continence before the procedure and the use of validated, self-administered evaluation means. Although technical improvements in all procedures should contribute to diminish the impact of complications, we should not forget the trend to the association of therapies- multimodal therapy- has a higher complication profile. Therefore, they should be reserved for patients in whom a benefit has been proved.

Keywords: Prostate cancer. Urinary incontinence. Epidemiology. Economics.

\section{INTRODUCCIÓN}

La glándula prostática por su localización entre ambos esfínteres urinarios e íntima relación con ellos juega un papel fundamental en la dinámica de la micción en el varón. Por ello patologías tanto ma- lignas como benignas de la misma interfieren con el patrón miccional. En el caso del cáncer de próstata (CaP), no solo el propio cáncer (en casos avanzados) puede afectar al proceso de la micción. Los procedimientos terapéuticos interfieren en la historia natural del $\mathrm{CaP}$, pero son también una importante fuente de morbilidad a largo plazo al afectar la continencia y la función eréctil. Este sucede con el tratamiento médico (el bloqueo hormonal) que entre otras cosas altera la dinámica de la relación entre estroma y epitelio de la glándula (ver capítulo previo). Sin embargo, son los procedimientos no médicos los que producen cambios mayores en el sistema esfinteriano y de la micción, entre ellos es bien conocida la afectación que puede producir la cirugía radical (prostatecomia radical -PR- abierta o laparoscópica). Otros procedimientos como la cirugía paliativa (RTU desobstructiva) o los tratamientos alternativos a la cirugía; la radioterapia externa, la braquiterapia, los ultrasonidos de alta-intensidad (high-intensity focused ultrasound -HIFU-) o la crioterapia también producen, en mayor o menor grado, disfunción en la esfera de la micción.

Los mecanismos por los que se produce la disfunción miccional y en concreto la incontinencia, son diversos y se detallan en otros capítulos de este monográfico. Probablemente los factores más determinantes son la edad y la calidad de la función miccional en el momento del procedimiento. En el caso de la cirugía radical, con la introducción de la técnica laparoscópica y las mejoras en las técnicas de imagen se conocen factores claves de estos procesos que incluyen; factores preoperatorios (edad y peso del paciente, actividad física-ejercicios pélvicos, volumen prostático), factores anatómicos (denervación trigonal, preservación de la fascia endopélvica, disección respetando músculo puboperineal) y factores técnicos (preservación del cuello vesical, de la longitud uretral, de las bandeletas neurovasculares y de los ligamentos puboprostáticos, así como la eversión mucosa) (1). La resonancia endorectal ha permitido recientemente la evaluación en profundidad de parámetros como son la longitud y la fibrosis uretral (2). Esta lesión uretral participa también en los trastornos de la continencia que afectan a los otros procedimientos como es el caso de la lesión por bajas temperaturas inducida por la crioterapia (3).

La definición de incontinencia empleada varia según las publicaciones, así como también sucede con el tiempo post procedimiento en que esta se evalúa y la manera de evaluarla (4). La ICS (Internacional Continente Society) ha intentado unificar criterios y definir como incontinencia "la queja de un escape involuntario de orina que puede producirse durante un esfuerzo, estornudo o tos (incontinencia de esfuerzo), acompañado o inmediatamente precedido de urgencia (incontinencia de urgencia), asociado a urgencia 
y también esfuerzo, estornudo o tos (incontinencia mixta)" $(5,6)$. Así algunos trabajos emplean la definición de "ausencia total de protectores" (7), mientras que otros describen las diversas frecuencias del uso de los mismos o cantidad de pérdida urinaria (8). Esto puede explicarse porque hasta el $13.4 \%$ de hombres sometidos a una PR presentan preoperatoriamente un cierto grado de escapes de orina (8), por lo que empleando parámetros estrictos de continencia deberían ser considerados incontinentes ya antes a la cirugía. Por ello algunos autores argumentan que es valido considerar como continentes a aquellos pacientes que emplean un solo protector al día o presentan algún mínimo escape ocasional (9).

Existen dos factores, a nuestro parecer de relevancia especial, cuando se evalúa la incontinencia. Por un lado el hecho de que el parámetro basal siempre debe ser el grado de continencia previa al procedimiento y que por tanto debería siempre documentarse antes del tratamiento. Por otro lado, el método de evaluación de la continencia debería, idealmente, ser autoadministrado. Litwin et al. (4), estudiando la base de datos del CAPSURE, demostraron diferencias importantes en la incidencia de la continencia (así como de la disfunción eréctil) dependiendo de si esta función era evaluada por el cirujano o por el paciente. Existe un cierto consenso de que la manera de evitar el sesgo inherente del cirujano, es que este no esté involucrado ni tenga una participación directa en la recogida de datos y que se emplee un instrumento (encuesta, cuestionario, etc) validado y auto-administrado por el paciente.

\section{INCIDENCIA}

La falta de uniformidad en la definición de incontinencia, la manera de evaluarla y la manera de reportarla hacen que la interpretación de los resultado funcionales tras cualquiera de los tratamientos establecidos para el CaP sea dispar y difícil de interpretar (10). A esto hay que añadir la variabilidad en la habilidad individual, en las preferencias del cirujano o radioterapeuta y en la selección de los pacientes. Aunque no existen estudios randomizados comparando los de diferentes técnicas, las series más actuales confirman la percepción de que la incontinencia urinaria post tratamiento afecta con mayor frecuencia a los pacientes tratados mediante cirugía radical.

Empleando encuestas de calidad de vida relativa a la salud realizadas antes del tratamiento y repetidas tras el mismo se evalúa no solo la incidencia de determinados parámetros sino también su evolución (mejora o empeoramiento) con el tiempo, así como su impacto sobre la calidad de vida. La recuperación de la incontinencia tras PR en series que emplean cuestio- narios autoadministrados ronda las cifras del $93 \%$ (de 3477 pacientes) (11) y del 90\% (de 1288 pacientes) (12). Una de las series norteamericanas más recientes (475 pac) publicada por Sanda et al. (13) observa que la incontinencia, a los 2 años del tratamiento, es más común tras prostatectomia radical que tras braquiterapia o irradiación externa (ambas $P<.001$ ) (14). El límite más o menos arbitrario de los 2 años de seguimiento se basa en principio en el concepto de que la mejora llega a un plateu tras ese periodo. Sin embargo, este límite ha sido recientemente puesto en duda al demostrarse que pueden producirse mejoras clínicamente significativas en el control urinario (así como en la función eréctil) más allá de los dos años tras la PR (9). En este trabajo se evaluó la continencia en 731 pacientes consecutivos sometidos a PR abierta; a los 2 años del procedimiento un $96.7 \%$ de los pacientes habían recuperado la continencia. Cuando los mismos pacientes fueron seguidos más allá de los dos años y durante un mínimo de 4 años, el $23 \%$ de los mismos manifestó la percepción de "algún grado de mejora" en la continencia siendo "moderada o marcada" en el $12.2 \%$. Por ello, los autores argumentan que es posible que pacientes etiquetados como "continentes" a los 2 años del procedimiento presenten con el tiempo una mejora de este parámetro. Estos resultados se contradicen con los del PCOS (Prostate Cancer Outcomes Study) en los que la incidencia de incontinencia aumentó del $10.4 \%$ al $13.9 \%$ entre los 2 y los 5 años de seguimiento, aunque esto podría explicarse por diferencias metodológicas y por el hecho de que el PCOS no partía de la continencia basal (15). Por otro lado, se han reportado excelentes resultados de continencia de hasta el 92\% en el grupo de pacientes sometidos a PR por $\mathrm{CaP}$ de alto riesgo o localmente avanzado (16).

En las series comparativas de la cirugía abierta vs. laparoscópica, los grados de continencia varían del 66.7 al $83.9 \%$ para la PR abierta y del 48 al $80.7 \%$ en la PR laparoscópica $(7,17)$. Las series más recientes sugieren que la preservación de bandeletas neurovasculares, tanto en la PR abierta (18) como en la laparoscópica (19), tiene un impacto positivo en la continencia. Asimismo se sabe que la incidencia de complicaciones se reduce si el procedimiento se lleva a cabo en hospitales con gran volumen de pacientes y por cirujanos con un elevado número de casos (20). El desarrollo de la PR robótica puede afectar el rendimiento global de la técnica quirúrgica y es en la actualidad sustrato de un profundo debate y de posiciones encontradas (21-24) como lo fue en su momento la aparición de la PR laparoscópica (7, 25-27). Aunque este debate trasciende los límites de esta revisión si apuntaremos que los datos más recientes en cirugía robótica, totalmente establecida ya en EE.UU., muestran resultados de función miccional al menos superponibles a los de la PR abierta e incluso con una más 
precoz recuperación de la continencia inmediata y a los 3 meses (24). Sin embargo no hay que olvidar que por el momento solo de dispone de información de series de casos, con la limitación que esto implica y con seguimientos a corto y medio plazo.

En la radioterapia externa se ha documentado el desarrollo de una incontinencia moderada (grado 2) o peor, como efecto adverso tardío, en el 5,3\% de pacientes tratados $(28,29)$. Este porcentaje es probable que disminuya con el uso de nuevas técnicas de radioterapia de intensidad modulada. Sin embargo la asociación frecuente de dolor rectal y diarrea, que llegan a desarrollar hasta el $40 \%$ de los pacientes, agrava la sintomatología miccional (29). El trabajo de Sanda et al., prospectivo y multiregional, evalúa la calidad de vida tras braquiterapia, radioterapia y prostatectomia (13). Al año de tratamiento el porcentaje de pacientes afectos de un grado moderado o severo de distress o síntomas urinarios fue del 18, 11 y $7 \%$ respectivamente. En el caso de la radioterapia radical, el estudio muestra una mejora de los resultados para sintomatología urinaria entre los 12 y los 24 meses y sostiene que cuando comparada prospectivamente tiene menos efectos negativos que los otras dos opciones de tratamiento.

Cuando la radioterapia se emplea como adyuvante a la cirugía en pacientes de alto riesgo, esta se asocia a unas tasas de incontinencia total superiores a las de los pacientes con los mismos factores de riesgo randomizados a recibir únicamente cirugía radical (30). Aunque la incontinencia total en esta serie de Bolla et al. sólo afectó al 6,5\% de los pacientes sometidos a RT adyuvante, hasta el $23,8 \%$ presentaron algún tipo de complicación. Esto es relevante porque aunque el estudio observó una reducción del riesgo de recurrencia y de fallo bioquímico, el tratamiento con radioterapia adyuvante no consiguió demostrar una mejora en la supervivencia global ni la supervivencia libre de metástasis. Las conclusiones de otras publicaciones son similares (30-32) y además, no todos los estudios han evaluado la incontinencia asociada al tratamiento (31). Algo similar sucede cuando la cirugía se emplea como rescate tras la radioterapia, observándose un incremento de la morbilidad con hasta un tercio de los pacientes presentando incontinencia o estenosis uretral (33).

La braquiterapia transperineal se asocia en especial a disfunción eréctil desarrollada a largo plazo así como a morbilidad rectal. Asimismo, la incidencia de incontinencia no es despreciable con afectación del 4 al $6 \%$ de los pacientes al año y dos años del tratamiento (13). En esta serie, además, la braquiterapia cursó también con una vitalidad reducida. Los datos de este estudio multicéntrico corroboran datos de otros centros en cuanto a la sintomatología asociada a la esfera de la función miccional así como de la intestinal y sexual para esta modalidad terapéutica $(19,34)$.

La incidencia de incontinencia asociada a la crioterapia varia entre el 1.3 y el $19 \%$ dependiendo de la definición empleada $(3,35)$ y con la limitación de que todos los estudios son series de casos y no hay estudios randomizados que comparen esta terapia como tratamiento primario del CaP localizado. Como con las otras opciones terapéuticas, las mejoras técnicas como el uso del calentamiento uretral y la monitorización de la temperatura de las criosondas ha permitido disminuir la incidencia de la incontinencia post procedimiento (3).

En el caso del HIFU como tratamiento del CaP se dispone de poca información con evidencia suficiente y no existen datos comparativos con otras técnicas ni datos claros sobre la incidencia de complicaciones y su impacto en la calidad de vida (36). Además parte de la series incluyen casos en los que esta se emplea como rescate tras la radioterapia externa por lo que la incidencia de la incontinencia es más difícil de valorar (36).

\section{CALIDAD DE VIDA}

El diagnostico de un cáncer es para el paciente lo que en ingles llaman "a life changing event". El CaP suele progresar lentamente y prácticamente el $100 \%$ de los pacientes tratados por un CaP localizado están vivos los 5 años del diagnostico (28). Por ello, los efectos adversos potenciales y las complicaciones a largo plazo son factores críticos a la hora de seleccionar las opciones de tratamiento. Las consecuencias del tratamiento y sus implicaciones suelen infravalorarse antes de someterse a tratamiento (37). Tras el mismo, los fenómenos de la incontinencia y la disfunción eréctil empiezan a tener más importancia, no solo para el paciente sino también para su pareja (13). El gran impacto que el tratamiento tiene en estas esferas de la vida de relación, la continencia y la sexualidad, hace que actualmente se promueva el objetivo no solo de conseguir el control del cáncer sino también el de la continencia y la potencia; lo que se llama resultados "trifecta" y que en las mejores series de PR se logra en aproximadamente dos tercios de los casos (38).

Cada modalidad de tratamiento para el CaP se asocia a un patrón distinto de cambios en los dominios de calidad de vida relacionados con la función urinaria, sexual, intestinal y hormonal. Estos cambios influyen en la satisfacción y la apreciación de los "resultados" del procedimiento (13). Sin embargo, es especialmente relevante el hecho de que las características psicosociales de base, tales como los síntomas depresivos, son predictores de los parámetros de cali- 
dad de vida post tratamiento, tanto o incluso más que la modalidad terapéutica (39).

Los dos principales estudios de calidad de vida, ambos prospectivos (no-randomizados) (13, 39) comparan los distintos patrones de cambios en la calidad de vida relacionados con cada modalidad terapéutica; PR, RT externa o braquiterapia, (aunque en el caso del estudio de Eller et al. las modalidades de RT fueron; RT intensidad modulada+braquiterapia permanente y RT intensidad modulada+RTaltas dosis -braquiterapia temporal-). Los pacientes sometidos a uno $u$ otro tratamiento son diferentes en cuanto a diversos parámetros que luego influyen en la calidad de vida y las diferencias no se refieren únicamente al estadio de la enfermedad. Así en la serie de Sanda et al. había mas enfermedades coexistentes en el grupo de pacientes tratados con radioterapia, seguido de aquellos tratados mediante braquiterapia, mientras que los pacientes sometidos a PR conformaban el grupo con menos enfermedades asociadas. La severidad del cáncer, en cambio, era mayor en el grupo sometido a radioterapia, intermedio en el de Prad y menor en el de los pacientes tratados con braquiterapia. Otro estudio importante es el del Prostate Cancer Outcomes Study (PCOS) (15), aunque en este caso se recogió la información pretratamiento retrospectivamente y no se estudió el efecto de la braquiterapia. También son limitados los datos del CaPSURE (Cancer of the Prostate Strategic Urologic Research Endeavor) porque no se evaluaron síntomas relacionados con la clínica irritativa miccional ni con el tratamiento hormonal (40).

Las conclusiones más relevantes de estos estudios, además de la mayor incidencia de incontinencia en los pacientes tratados mediante cirugía, incluyen la observación de que la braquiterapia se asocia también a incontinencia y a disminución de la vitalidad. Asimismo se ha observado un papel importante de la técnica robótica en unos mejores resultados físicos y sobretodo una recuperación más rápida que los pacientes operados por la técnica de cirugía abierta. Cuando se evaluó la vivencia de la pareja; el $5 \%$ de las parejas de pacientes sometidos a PR se mostraron molestas por la incontinencia. En cambio en las técnicas de irradiación la experiencia de la pareja mostró también molestias por cambios en la esfera de la micción pero en este caso por síntomas de obstrucción urinaria en el $7 \%$ de aquellas sometidos a braquiterapia y el $3 \%$ de los sometidos a radioterapia. Otras series también observan que los síntomas irritativos y obstructivos son significativamente más prevalentes tras braquiterapia que tras $\operatorname{PR}(14,19)$. Además, aunque en líneas generales el control urinario y la función sexual obtienen mejores resultados tras radioterapia externa, la cirugía con preservación de bandeletas ha demostrado disminuir estas diferencias cuando se parte de pacientes potentes pre PR (19).
De modo similar, en nuestro medio, un estudio prospectivo de 304 pacientes sometidos a diferentes modalidades de tratamiento radical (PR, irradiación externa $\circ$ braquiterapia) ha comparado los efectos de cada uno de ellos en la calidad de vida. Se emplearon, entre otros, el cuestionario de salud SF-36 y el Expanded Prostate Cancer Index Composite (EPIC), administrados antes y después del tratamiento a intervalos periódicos (41). En todos los casos los valores se recuperaron parcialmente con el tiempo después de un deterioro inicial siendo la incontinencia urinaria una complicación más evidente en los pacientes sometidos a PR. Sin embargo, como en otras series, los pacientes tratados con braquiterapia mostraron un deterioro de los síntomas obstructivos que también es destacable a la hora de comparar efectos sobre la calidad de vida.

Otros factores de impacto en la calidad de vida son, por ejemplo, en el caso de la radioterapia externa la asociación frecuente de sintomatología rectal, con hasta un $39 \%$ de los pacientes presentando dolor rectal el primer año tras el procedimiento (28). En el caso de la braquiterapia ya hemos mencionado el papel de los síntomas irritativos. Con la crioterapia, a la incontinencia y las molestias uretrales hay que añadir la disfunción eréctil que afecta al a mayoría de pacientes con solo del 5 al $47 \%$ reportando evidencias de mejora (3). Para todas las modalidades de tratamiento, las mejoras técnicas deberían contribuir a disminuir el impacto de las complicaciones. Sin embargo, no hay que olvidar la tendencia a asociar tratamientos con el riesgo de empeorar los efectos secundarios que conlleva. Las terapias multimodales, en especial la asociación de RT externa con braquiterapia, pero también la adición de hormonoterapia, tienen un perfil de complicaciones mas elevado, por lo que deben reservarse para aquellos pacientes en los que se haya demostrado un beneficio (28).

\section{ASPECTOS FARMACOECONÓMICOS}

El tratamiento del CaP y de sus complicaciones además de ser una carga emocional sustancial para los pacientes y para sus pareja/familias, también lo es para el sistema sanitario y socioeconómico. Los aspectos farmacoeconómicos son una consideración importante a tener en cuenta cuando se evalúan los efectos del CaP y de sus complicaciones en la sociedad. Como sucede con los parámetros de calidad de vida, existe una gran variabilidad en la maneras de medir los costes que además esta influida por el tipo de sistema sanitario de cada país. Por ejemplo, en EEUU el coste por paciente los primeros 6 meses tras el diagnóstico de CaP es de unos 11.500 \$ USA (28). Otros parámetros que pueden emplearse son, por ejemplo, los costes por persona a lo largo de 15 años. 
Este tipo de evaluación acumulativa de costes permite una estimación más completa de los gastos generados por cada tipo de tratamiento. En estos casos se contemplan no solo los gastos del procedimiento inicial sino también los del impacto social de la baja del paciente así como los gastos generados por el seguimiento y tratamiento de las complicaciones y del tratamiento de la recurrencia de la enfermedad. Estas evaluaciones son frecuentes en EEUU pero no en nuestro medio y para ello disponemos de poca información exhaustiva y de calidad.

Existen también comparaciones de costes entre las diferentes técnicas de tratamiento (42). Por ejemplo la crioterapia es un procedimiento que tiene la ventaja de no requerir de un equipo caro, como sucede con la radioterapia o la PR robótica, aunque la curva de aprendizaje es larga. Algún estudio sugiere que es un procedimiento mas barato que la cirugía radical, aunque solo han evaluado los costes hospitalarios a corto plazo (3).

En cuanto a los gastos asociados a la incontinencia, estos incluyen desde las visitas médicas y las exploraciones urodinámicas, hasta el uso de protectores y el tratamiento médico o quirúrgico final. No disponemos de mucha información detallada en nuestro medio de estos costes, pero si sabemos lo que cuesta el tratamiento del CaP en países como EEUU o el coste de un esfínter artificial o un sling transobturador. Por otro lado datos recientes reflejan un incremento de los gastos asociados al tratamiento de canceres en estadio inicial (incluyendo mama, pulmón, colon y próstata) (43). Esto refleja el acceso a tratamiento por parte de más pacientes, pero también un incremento de los precios de estos tratamientos y de sus potenciales complicaciones.

\section{BIBLIOGRAFÍA y LECTURAS RECOMENDADAS (*lectura de interés $y^{* *}$ lectura fundamental)}

*1. Cambio AJ, Evans CP. Minimising postoperative incontinence following radical prostatectomy: considerations and evidence. Eur Urol 2006; 50(5):903-13; discussion 913.

**2. Paparel P, Akin O, Sandhu JS, Otero JR, Serio AM, Scardino PT, et al. Recovery of urinary continence after radical prostatectomy: Association with urethral length and urethral fibrosis measured by preoperative and postoperative endorectal magnetic resonance imaging. Eur Urol 2009;55:629-39.

3. Shelley M, Wilt TJ, Coles B, Mason MD. Cryotherapy for localised prostate cancer. Cochrane Database Syst Rev 2007; (3):CD005010.

*4. Litwin MS, Lubeck DP, Henning JM, Carroll PR. Differences in urologist and patient assessments of health related quality of life in men with pros- tate cancer: results of the CaPSURE database. J Urol 1998; 159(6):1988-92.

5. Abrams P, Artibani W, Cardozo L, Dmochowski R, van Kerrebroeck P, Sand P. Reviewing the ICS 2002 terminology report: the ongoing debate. Neurourol Urodyn 2009; 28(4):287.

6. Abrams P, Cardozo L, Fall M, Griffiths D, Rosier $\mathrm{P}$, Ulmsten U, et al. The standardisation of terminology in lower urinary tract function: report from the standardisation sub-committee of the International Continence Society. Urology 2003; 61(1):37-49.

7. Touijer K, Eastham JA, Secin FP, Romero Otero J, Serio A, Stasi J, et al. Comprehensive prospective comparative analysis of outcomes between open and laparoscopic radical prostatectomy conducted in 2003 to 2005. J Urol 2008; 179(5):1811-7; discussion 1817.

8. Lepor H, Kaci L. The impact of open radical retropubic prostatectomy on continence and lower urinary tract symptoms: a prospective assessment using validated self-administered outcome instruments. J Urol 2004; 171(3):1216-9.

*9. Glickman L, Godoy G, Lepor H. Changes in continence and erectile function between 2 and 4 years after radical prostatectomy. J Urol 2009; 181(2):731-5.

10. Corcos J, Beaulieu S, Donovan J, Naughton M, Gotoh M. Quality of life assessment in men and women with urinary incontinence. J Urol 2002; 168(3):896-905.

**11. Kundu SD, Roehl KA, Eggener SE, Antenor JA, Han M, Catalona WJ. Potency, continence and complications in 3,477 consecutive radical retropubic prostatectomies. J Urol 2004; $172(6 \mathrm{Pt}$ 1):2227-31.

*12. Penson DF, McLerran D, Feng Z, Li L, Albertsen PC, Gilliland FD, et al. 5-year urinary and sexual outcomes after radical prostatectomy: results from the prostate cancer outcomes study. J Urol 2005; 173(5):1701-5.

**13. Sanda MG, Dunn RL, Michalski J, Sandler HM, Northouse L, Hembroff L, et al. Quality of life and satisfaction with outcome among prostate-cancer survivors. N Engl J Med 2008; 358(12):1250-61.

14. Gore JL, Kwan L, Lee SP, Reiter RE, Litwin MS. Survivorship beyond convalescence: 48-month quality-of-life outcomes after treatment for localized prostate cancer. J Natl Cancer Inst 2009; 101(12):888-92.

*15. Penson DF, McLerran D, Feng Z, Li L, Albertsen PC, Gilliland FD, et al. 5-year urinary and sexual outcomes after radical prostatectomy: results from the Prostate Cancer Outcomes Study. J Urol 2008; 179(5 Suppl):S40-4.

16. Loeb S, Smith ND, Roehl KA, Catalona WJ. Intermediate-term potency, continence, and survival outcomes of radical prostatectomy for clinically high-risk or locally advanced prostate cancer. Urology 2007;69(6):1170-5.

17. Romero Otero J, Martinez-Salamanca JI. Critical comparative analysis between open, laparoscopic 
and robotic radical prostatectomy: urinary continence and sexual function (part II). Arch Esp Urol 2007; 60(7):767-76.

*18. Montorsi F, Salonia A, Suardi N, Gallina A, Zanni $\mathrm{G}$, Briganti A, et al. Improving the preservation of the urethral sphincter and neurovascular bundles during open radical retropubic prostatectomy. Eur Urol 2005;48(6):938-45.

**19. Litwin MS, Gore JL, Kwan L, Brandeis JM, Lee SP, Withers HR, et al. Quality of life after surgery, external beam irradiation, or brachytherapy for early-stage prostate cancer. Cancer 2007; 109(11):2239-47.

20. Begg CB, Riedel ER, Bach PB, Kattan MW, Schrag D, Warren JL, et al. Variations in morbidity after radical prostatectomy. N Engl J Med 2002; 346(15):1138-44.

21. Blute ML. Radical prostatectomy by open or laparoscopic/robotic techniques: an issue of surgical device or surgical expertise? J Clin Oncol 2008; 26(14):2248-9.

22. Hu JC, Wang Q, Pashos CL, Lipsitz SR, Keating NL. Utilization and outcomes of minimally invasive radical prostatectomy. J Clin Oncol 2008; 26(14):2278-84.

23. Blute ML. No proof of inferiority: open radical retropubic prostatectomy remains state-of-the-art surgical technique for localized prostate cancer. $\mathbf{J}$ Urol 2009; 181(6):2421-3.

24. Pruthi RS, Wallen EM. Current status of robotic prostatectomy: promises fulfilled. J Urol 2009; 181(6):2420-1.

*25. Romero-Otero J, Touijer K, Guillonneau B. Laparoscopic radical prostatectomy: contemporary comparison with open surgery. Urol Oncol 2007; 25(6):499-504.

26. Guazzoni G, Cestari A, Naspro R, Riva M, Centemero A, Zanoni M, et al. Intra- and peri-operative outcomes comparing radical retropubic and laparoscopic radical prostatectomy: results from a prospective, randomised, single-surgeon study. Eur Urol 2006; 50(1):98-104.

27. Rassweiler J. Open vs. laparoscopic radical prostatectomy. and laparoscopy is better! Eur Urol 2006; 50(1):26-8.

28. Gomella LG, Johannes J, Trabulsi EJ. Current prostate cancer treatments: effect on quality of life. Urology 2009; 73(5 Suppl):S28-35.

29. Thompson I, Thrasher JB, Aus G, Burnett AL, Canby-Hagino ED, Cookson MS, et al. Guideline for the management of clinically localized prostate cancer: 2007 update. J Urol 2007; 177(6):2106-31.

30. Thompson IM, Jr., Tangen CM, Paradelo J, Lucia MS, Miller G, Troyer D, et al. Adjuvant radiotherapy for pathologically advanced prostate cancer: a randomized clinical trial. Jama 2006; 296(19):2329-35.

*31. Bolla M, van Poppel H, Collette L, van Cangh P, Vekemans K, Da Pozzo L, et al. Postoperative radiotherapy after radical prostatectomy: a randomised controlled trial (EORTC trial 22911). Lancet $2005 ; 366(9485): 572-8$.
*32. Wiegel T, Bottke D, Steiner U, Siegmann A, Golz $\mathrm{R}$, Storkel S, et al. Phase III postoperative adjuvant radiotherapy after radical prostatectomy compared with radical prostatectomy alone in pT3 prostate cancer with postoperative undetectable prostate-specific antigen: ARO 96-02/AUO AP 09/95. J Clin Oncol 2009; 27(18):2924-30.

33. Stephenson AJ, Eastham JA. Role of salvage radical prostatectomy for recurrent prostate cancer after radiation therapy. J Clin Oncol 2005; 23(32):8198-203.

34. Brandeis JM, Litwin MS, Burnison CM, Reiter RE. Quality of life outcomes after brachytherapy for early stage prostate cancer. J Urol 2000;163(3):851-7.

35. El Hayek OR, Alfer W, Jr., Reggio E, Pompeo AC, Arap S, Lucon AM, et al. Prostate cryoablation: prospective analysis comparing high- and low-risk prostate cancer outcomes. Urol Int 2008; 81(2):186-90.

36. Lledo Garcia E, Jara Rascon J, Subira Ros D, Herranz Amo F, Martinez-Salamanca JI, Hernandez Fernandez C. Scientific evidence on the use of high-intensity focal ultrasound (HIFU) in the treatment of prostatic carcinoma. Actas Urol Esp 2005; 29(2):131-7.

*37. Zeliadt SB, Ramsey SD, Penson DF, Hall IJ, Ekwueme DU, Stroud L, et al. Why do men choose one treatment over another? a review of patient decision making for localized prostate cancer. Cancer 2006; 106(9):1865-74.

38. Bianco FJ, Jr., Scardino PT, Eastham JA. Radical prostatectomy: long-term cancer control and recovery of sexual and urinary function ("trifecta"). Urology 2005; 66(5 Suppl):83-94.

39. Eller LS, Lev EL, Gejerman G, Colella J, Esposito M, Lanteri V, et al. Prospective study of quality of life of patients receiving treatment for prostate cancer. Nurs Res 2006; 55(2 Suppl):S28-36.

40. Downs TM, Sadetsky N, Pasta DJ, Grossfeld GD, Kane CJ, Mehta SS, et al. Health related quality of life patterns in patients treated with interstitial prostate brachytherapy for localized prostate cancer--data from CaPSURE. J Urol 2003; 170(5):1822-7.

41. Guedea F, Ferrer M, Pera J, Aguilo F, Boladeras A, Suarez JF, et al. Quality of life two years after radical prostatectomy, prostate brachytherapy or external beam radiotherapy for clinically localised prostate cancer: the Catalan Institute of Oncology/Bellvitge Hospital experience. Clin Transl Oncol 2009; 11(7):470-478.

42. Wilson LS, Tesoro R, Elkin EP, Sadetsky N, Broering JM, Latini DM, et al. Cumulative cost pattern comparison of prostate cancer treatments. Cancer 2007;109(3):518-27.

43. Warren JL, Yabroff KR, Meekins A, Topor M, Lamont EB, Brown ML. Evaluation of trends in the cost of initial cancer treatment. J Natl Cancer Inst 2008; 100(12):888-97. 